\title{
Correcting acoustic measurements of scatterer density for extinction
}

\author{
Kenneth G. Foote \\ Institute of Marine Research, 5024 Bergen, Norway
}

(Received 8 March 1990; accepted for publication 31 May 1990)

\begin{abstract}
Extinction is sometimes a major problem in acoustic surveys of fish stocks, as it often occurs when the fish are concentrated and easiest to survey. The same may be true of certain macrozooplankton, such as krill in swarms. This study aims to describe how to correct singleping measurements of the vertical distribution of scatterer density for extinction. The general case is considered in which the aggregation density is variable and the mean backscattering and extinction cross sections vary with depth. By dividing the water column into a finite number of layers, with constant properties within each, a closed-form mean-field solution is derived.

Methods of applying this to single-ping echo records and the quality of the solution are both examined. Extinction is discussed vis-à-vis multiple scattering. Application of the technique in other areas, e.g., in remote probing of the atmosphere by lidar, is mentioned.
\end{abstract}

PACS numbers: 43.30.Xm, 43.20.Fn, 43.60.Pt

\section{INTRODUCTION}

In the last several fishing seasons, the all-important 1983 year class of Norwegian spring-spawning herring ( $\mathrm{Clu}$ pea harengus), on which the future of the stock depends, ${ }^{1,2}$ has been decimated by disastrously large catches that have literally burst nets. Such lost catches are not counted in the present quota system, and hence represent a pure, if inadvertent, overexploitation of the stock. Awareness of the need for caution in catching operations explains the following radio. conversation, overheard between two fishing vessels in a northern Norwegian fjord in December 1989. First fisherman: "Is it safe to fish here?" Second fisherman: "Yes; we can see the bottom."

That is, the second fisherman has concluded from the appearance of the echogram that the likely catch size will be manageable. Because the bottom echo signal could be "seen," or discerned, the column density of fish was not considered excessive for purse seining. Evidently, it is the fisherman's experience that fishing on an aggregation of herring that blocks or extinguishes the bottom echo signal is risky!

The phenomenon of extinction by fish aggregations is also of practical importance to researchers who must estimate fish abundance by acoustic means. ${ }^{3}$ This has been recognized in a number of experimental studies, ${ }^{4-11}$ which have sought to measure the effect of extinction or the extinction cross section. It has also been considered in several theoretical studies. ${ }^{3,12-14}$

Extinction may also be of interest in scattering by plankton. While this is undoubtedly negligible for phytoplankton, such as the unicellular green algae scenedesmus and diatom Nitzschia closterium $f$ minutissima, ${ }^{15}$ whether singly or in colonies, and some zooplankton, it may be quite important for macrozooplankton. Examples include Antarctic krill (Euphausia superba), observed by underwater divers in swarms with densities estimated to be of the order of 20000 60000 animals $/ \mathrm{m}^{3}$ (Ref. 16), and the krill Meganyctiphanes norvegica, which was recently observed acoustically in layers over $50 \mathrm{~m}$ thick and with an average density of 800 animals/ $\mathrm{m}^{3}$ (Ref. 17). Even at these densities the effect may not be large, and second-order scattering effects may be significant. ${ }^{18}$ Whether dense and extended aggregations of softbodied scatterers, such as jellyfish or salps, extinguish sound measurably, or produce significant echoes for that matter, is unknown. ${ }^{19}$

The effect of extinction has been described in several places for constant-density scattering layers. ${ }^{3,13,14}$ It may be thought that the generalization to variable-density layers is straightforward. However, there seems to be some confusion about this, at least in fisheries acoustics. In addition, for studies of structure in scatterer aggregations, such as fish schools, it is desired to describe the vertical distribution of density as sensed by single pings.

It is the present aim to extend the theory for constant density to variable density, and show how to correct singleping measurements of the vertical distribution of scatterer density for extinction.

\section{THEORY}

Several different quantities are suitable for describing acoustic measures of scatterer density. In Ref. 13, echo energy is used. The area or column backscattering coefficient ${ }^{20}$ or strength ${ }^{21}$ is the most widely used today. Since the present object is correction of density estimates for extinction in a variable-density layer, the derivative of the area backscattering coefficient with respect to depth is most convenient. This is just the volume backscattering coefficient. ${ }^{22}$ For a scatterer aggregation of constant number density $\rho$ and mean backscattering cross section $\sigma_{b}$, the volume backscattering coefficient in the neglect of extinction is

$$
s_{v}=\rho\left(\sigma_{b} / 4 \pi\right) \text {. }
$$

That is, the measured quantity $s_{v}$ is directly proportional to the scatterer density. This equation applies strictly only in the mean of a large number of independent observations on the same aggregation or, to a good approximation, to a single observation when the number of observed scatterers in the beam is sufficiently large. Assumptions of stationarity and sufficient numbers of scatterers are thus implicit in the use of 
Eq. (1) in this study. The theory consequently belongs to the category called "mean-field."

For a scattering layer of constant density $\rho$ and constant $\sigma_{b}$ over the depth range $\left[z_{1}, z_{2}\right], s_{v}$ is generally less than that indicated in Eq. (1). The degree of reduction is determined by integrating the steadily decreasing mean echo intensity, after the usual " $20 \log r+2 \alpha r$ " type of range compensation for a scattering layer, ${ }^{3,23}$ over the interval $\left[z_{1}, z_{2}\right]$. If there are no scatterers in the interval $\left[0, z_{1}\right], s_{v}$ for the scattering layer is thus modified by the single multiplicative factor

$$
\int_{z_{1}}^{z_{2}} \exp \left[-2 \rho \sigma_{e}\left(\zeta-z_{1}\right)\right] d \zeta /\left(z_{2}-z_{1}\right),
$$

where $\sigma_{e}$ is the mean extinction cross section. Here and elsewhere in this study, beam spreading is ignored. The incurred approximation is excellent for echo sounders using ordinary narrow beams, typically of the order of $8 \mathrm{deg}$ or less as measured between opposite - 3-dB levels. Integrating, then,

$$
s_{v}=\rho \frac{\sigma_{b}}{4 \pi} \frac{1-\exp \left[-2 \rho \sigma_{e}\left(z_{2}-z_{1}\right)\right]}{2 \rho \sigma_{e}\left(z_{2}-z_{1}\right)} .
$$

This result is consistent with the various formulas given in the cited theoretical studies. It also agrees with the analogous expression used in studies of backscattering of light by aerosols. ${ }^{24}$

In general, scatterer density does vary with depth in an aggregation. The mean quantities $\sigma_{b}$ and $\sigma_{e}$ may also vary with depth. To lessen the chances of confusion in addressing the general case, extinction is treated in elementary fashion.

The intensity of a plane acoustic wave incident on a scattering aggregation diminishes, in the mean, with depth of penetration into the aggregation. To first order, the relative change in intensity $\Delta I / I$ over the depth range from $z$ to $z+\Delta z$ is linearly proportional to the scatterer density $\rho$ and mean extinction cross section $\sigma_{e}$ characterizing the depth interval, and the interval thickness $\Delta z$ :

$$
\Delta I / I=-\rho \sigma_{e} \Delta z .
$$

Since this applies for the arbitrary interval, at any $z$, it can be integrated over $[0, z]$ :

$$
I(z)=I(0) \exp \left(-\int_{0}^{z} \rho(\zeta) \sigma_{e}(\zeta) d \zeta\right),
$$

where $I(0)$ is the effective incident intensity at the reference depth $z=0$, and the general depth dependences of the several factors in Eq. (3) are shown explicitly.

The volume backscattering coefficient at $z$, shown in Eq. (1), is thus diminished, in the mean, by the square of the exponential factor in Eq. (4), hence

$$
s_{v}(z)=\exp \left(-2 \int_{0}^{z} \rho(\zeta) \sigma_{e}(\zeta) d \zeta\right) \rho(z) \frac{\sigma_{b}(z)}{4 \pi} .
$$

The represented function is continuous. In practice, $s_{v}$ is sampled at discrete depths $\left\{z_{j}\right\}$ and either represented as such or, more often, averaged over subsets of the points, spanning small or large intervals. For this second case of averaging over a finite interval, say from $z_{1}$ to $z_{2}, s_{v}(z)$ in Eq. (5) should be replaced by the expression

$$
\begin{aligned}
s_{v}\left(z_{1}, z_{2}\right)= & \exp \left(-2 \int_{0}^{z_{1}} \rho(\zeta) \sigma_{e}(\zeta) d \zeta\right) \\
& \times \int_{z_{1}}^{z_{2}} \rho(z) \frac{\sigma_{b}(z)}{4 \pi} \\
& \times \exp \left[-2 \rho(z) \sigma_{e}(z)\left(z-z_{1}\right)\right] d z /\left(z_{2}-z_{1}\right),
\end{aligned}
$$

where the applicable interval of integration is indicated explicitly in the arguments of $s_{v}$. For the special case of a single scattering layer confined to the depth interval $\left[z_{1}, z_{2}\right]$, in which $\rho, \sigma_{b}$, and $\sigma_{e}$ are constant, Eq. (6) reduces to Eq. (2).

To compare Eqs. (5) or (6) with Eq. (34) in Ref. 3, the observational quantity $s_{v}$ here may be replaced by the product of an apparent density and $\sigma_{b} / 4 \pi$, according to Eq. (1), and $\sigma_{b}(z)$ and $\sigma_{e}(z)$ may be replaced by constants. Neither of the resulting equations is equivalent to the expression in Ref. 3, although purporting to describe the same, unknown density.

\section{SOLUTION}

The echo integration method aims to relate an acoustic measurement, such as that of the volume backscattering coefficient, to the causative scatterer density, given knowledge of the scatterer type. In the case of negligible extinction, $s_{v}$ and $\rho$ are proportional, and solution of Eq. (1) for $\rho$ is immediate. In the presence of extinction, and with ordinary discrete sampling, Eq. (6) must be solved. Several approaches are possible. The one presented here is the more heuristic.

The scatterer aggregation is assumed, for generality in analysis, to be divided into $N$ layers between the depths $z_{1}$ and $z_{N+1}$, which may represent the surface and bottom, respectively. The depth limits of the $j$ th layer are $z_{j}$ and $z_{j+1}=z_{j}+\Delta z_{j}$, where $\Delta z_{j}$ is the thickness of the $j$ th layer. Each layer thickness is chosen to be sufficiently small so that $\rho, \sigma_{b}$, and $\sigma_{e}$ may be assumed to be constant in the particular layer, with mean values $\rho_{j}, \sigma_{b j}$, and $\sigma_{e, j}$. The volume backscattering coefficient is similarly distinguished by the depth of sampling, viz. $s_{v, j}$.

Equation (6) is evaluated for the $n$th layer, with limits $z_{n}$ and $z_{n+1}$. The first integral, after redefinition of its limits as $z_{1}$ and $z_{n}$, can be written as

$$
\exp \left(-2 \sum_{j=1}^{n-1} \rho_{j} \sigma_{e j} \Delta z_{j}\right),
$$

with the implicit value of unity understood for $n=1$. By assumption of constant values $\rho_{n}, \sigma_{b, n}$, and $\sigma_{e, n}$ for the $n$th layer, the second integral is just

$$
(1 / 8 \pi)\left(\sigma_{b, n} / \sigma_{e, n}\right)\left[1-\exp \left(-2 \rho_{n} \sigma_{e, n} \Delta z_{n}\right)\right] .
$$

Combining the several results,

$$
\begin{aligned}
s_{v, n}= & \left(1 / 8 \pi \Delta z_{n}\right)\left(\sigma_{b, n} / \sigma_{e, n}\right)\left[1-\exp \left(-2 \rho_{n} \sigma_{e, n} \Delta z_{n}\right)\right] \\
& \times \exp \left(-2 \sum_{j=1}^{n-1} \rho_{j} \sigma_{e, j} \Delta z_{j}\right) .
\end{aligned}
$$

The solution for $\rho_{n}$ is immediate. Defining

$$
\hat{s}_{v, n}=s_{v, n} \exp \left(2 \sum_{j=1}^{n-1} \rho_{j} \sigma_{e, j} \Delta z_{j}\right) .
$$




$$
\rho_{n}=-\frac{1}{2 \sigma_{e, n} \Delta z_{n}} \ln \left(1-\frac{8 \pi \hat{s}_{v, n} \Delta z_{n} \sigma_{e, n}}{\sigma_{b, n}}\right)
$$

With the aforementioned understanding that the exponential term in Eq. (8a) is unity for $n=1, \rho_{n}$ is evaluated in successive order from $n=1$ to $n=N$.

The solution given in Eq. (8) is generally well-behaved. In the limit that $\Delta z_{n}$ or $\sigma_{e, n}$ vanishes, $\rho_{n}=4 \pi \hat{s}_{v, n} / \sigma_{b, n}$, which is better interpreted as the result for an acoustically thin layer, by analogy with optics. For an empty layer, with $s_{v, n}=0, \rho_{n}=0$. Layers with few scatterers are subject to larger fluctuations in $s_{v, n}$ than those with many scatterers, but the influence of fluctuations in the first $n-1$ layers on $\rho_{n}$ is dampened by the manner of combination of the $n-1$ density estimates in Eq. (8a).

\section{DISCUSSION}

\section{A. Applying the solution}

Measurements of scatterer density may be corrected for extinction according to Eq. (8). This may be done for a series of layers of arbitrary thickness, as long as $\rho, \sigma_{b}$, and $\sigma_{e}$ remain essentially constant in each. A practicable scheme for ensuring this in ordinary survey work entails choosing layers of equal thickness $\Delta z=1 \mathrm{~m}$. This is also a very common unit of resolution in digital representations of $s_{v}$.

Application of the formula in real time, based on prior assumption of the kind of scatterer, hence $\sigma_{b}$ and $\sigma_{e}$, is also possible. Such adaptive processing would, however, make excessive demands on even the latest-generation scientific echo sounding and integration system, ${ }^{25}$ and is moreover unnecessary because of the ease and speed of postprocessing by means of workstation-level computers. ${ }^{26,27}$

A particular advantage of the formula is that it can be executed without further operator assistance once the scatterer is classified. This initial operator-determined process consists of specification of scatterer identity, namely species and size distribution, hence mean backscattering and extinction cross sections, with or without specified depth dependences, in the case of a single-species aggregation. In the case of a mixed aggregation or multiple scattering layers, the species content, several size distributions, and respective depth ranges must also be specified. Notwithstanding the apparent magnitude and difficulty of this assignment, it is nothing more than is done every time that an echogram is interpreted for the purpose of estimating fish density along an acoustic survey track. The single new element in the formula is the mean extinction cross section, but this is known for some species, ${ }^{7,8,12}$ and is being ${ }^{11}$ or will be specified for others. As noted by MacLennan et al., ${ }^{11} \sigma_{e}$ will undoubtedly vary with fish behavior and physiological condition, but then so does $\sigma_{b}$, and to a greater degree too, since the extent of the averaging implicit in $\sigma_{e}$ exceeds that in $\sigma_{b}$.

A second advantage of the formula is that it subsumes arbitrary density variations or fluctuations. A scattering layer need not be stratified vertically, but may vary in any or all directions.

\section{B. Quality of the solution}

The numerical error incurred in evaluating Eq. (8a) is readily defined by a simple analysis. If the formula is effected on a digital computer with a single-precision floating-point word size that allots $m$ bits to the mantissa, exclusive of the sign bit, then the numerical error $\epsilon_{j}$ in the density estimate $\rho_{j}$ will lie in the range $[-0.5,0.5]$ of the last or $m$ th bit. A more complete representation of a single component of the exponential factor in Eq. (8a) is $\exp \left[x_{j}\left(1+\epsilon_{j} / \rho_{j}\right)\right]$ or $\exp \left(x_{j}+\delta_{j}\right)$, where $\delta_{j}=x_{j} \epsilon_{j} / \rho_{j}=2 \epsilon_{j} \sigma_{e j} \Delta z_{j}$. Since $\delta_{j}$ is generally quite small, the following Maclaurin expansion is appropriate:

$$
\exp \left(\delta_{j}\right)=1+\delta_{j}+\left(\delta_{j}^{2} / 2\right)+\cdots,
$$

hence, the relative cumulative error in, say, $\rho_{n+1}$ due to the exponential factor in Eq. (8a) is

$$
\begin{aligned}
\exp \left(\sum_{j=1}^{n} \delta_{j}\right)-1= & \sum_{j=1}^{n} \delta_{j}+\frac{1}{2} \sum_{j=1}^{n} \delta_{j}^{2} \\
& +\sum_{j \neq k}^{n} \sum_{j \neq k}^{n} \delta_{j} \delta_{k}+O\left(\delta_{j} \delta_{k} \delta_{l}\right) .
\end{aligned}
$$

If there are $N$ contributing terms in the summation, then the worst-case error is $N\left|\delta_{j}\right|_{\max }=N \cdot 2\left|\epsilon_{j} \sigma_{e, j} \Delta z_{j}\right|_{\max }$ $=N\left(\sigma_{e, j} \Delta z_{j}\right)_{\max } / 2^{m}$. A more realistic analysis assumes that the errors are independent and uniformly distributed on the interval $[-0.5,0.5]$ of the $m$ th bit, with variance $1 / 12$. By the central limit theorem, their sum over a sufficiently large number of samples will be normally distributed. Thus the relative cumulative error will be less than $2.88(N / 12)^{1 / 2}\left(\sigma_{e, j} \Delta z_{j}\right)_{\mathrm{rms}} / 2^{m}$ with $99.9 \%$ probability.

To judge the magnitude of the several errors described here, the case that $N=500$ and $m=23$ is considered. This corresponds to, for example, a 500-m depth range with depth resolution $\Delta z=1 \mathrm{~m}$ in $s_{v}$, and processing by the SUN-4 workstation series of digital computers, among other machines, with a 32-bit single-precision word size. If $\left(\sigma_{e, j} \Delta z_{j}\right)_{\max }$ were to equal unity in SI units, which is a gross overestimate, the worst-case relative cumulative error is of the order of $3 \times 10^{-5}$.

Clearly, the purely numerical error that can arise in effecting the formula will be negligible, at least for digital processing with ordinary 32-bit word sizes. Errors due to uncertainty in $\sigma_{b}, \sigma_{e}$, and their several depth dependences are more serious.

Fluctuations in the acoustic field from the assumed mean-field will also contribute to the overall error. Extinction by an ensemble of scatterers is a stochastic process, and the solution derived here is therefore applicable in a statistical sense, namely in the mean of a large number of observations or approximately in the presence of a large number of scatterers. Likely magnitudes of errors resulting from failure of the mean-field assumption can be investigated by simulation, although involving formidable numerical computations.

What is theoretically most hazardous is attempting to correct measurements of scatterer density when the effect of extinction is very large. In this case, which is fortunately expected to be very rare, evaluation of the formula must be 
halted well before the noise level is reached. As the noise level is approached, the uncertainty in density estimates, hence also in the extinction-adjusted estimates $\hat{s}_{v, n}$, must be very large. The damaging effect of an error in $\hat{s}_{v, n}$ on $\rho_{n}$ is apparent from Eq. (8b). Thus in the presumed case of very high extinction, an impasse will be met. Advances must then be sought in alternate instrumentation, e.g., a lower-frequency transducer.

\section{Multiple scattering}

The overall objective in correcting scatterer density estimates for extinction is to derive the best possible estimates. Thus multiple scattering also deserves to be considered. This was done by Stanton for second-order scattering by a random distribution of isotropic scatterers, yielding upper bounds for the effect. ${ }^{18}$ One conclusion of this study was that extinction would be the dominant effect if the degree of extinction were significant. Another conclusion was that the effect of second-order scattering is less for narrower transducer beams.

With respect to the envisaged particular application to dense and thick aggregations of herring, the degree of extinction is particularly large. Sometimes the bottom echo signal does disappear on the echogram, i.e., becomes indistinguishable from noise. Considering the geometric extent of typical fish aggregations, transducer beamwidths of survey use are very or extremely narrow by Stanton's criterion. Since nominal acoustic wavelengths, $1-4 \mathrm{~cm}$, are also much smaller than typical fish lengths, $30-35 \mathrm{~cm}$, and smaller than corresponding swimbladder lengths, approximately $10-12 \mathrm{~cm}$, the scattering is directional, or anisotropic, and second-order scattering effects are indeed expected to be quite small.

Interestingly, application of the extinction-correction algorithm to macrozooplankton may require careful attention to second- or even higher-order scattering. The reason is that the animals are smaller and their densities higher, and they are often surveyed at shorter ranges, hence with effectively wider-beam scattering geometries, according to Stanton. ${ }^{18}$ The literature review performed by this author, in $1982,{ }^{28}$ supports Stanton's more detailed conclusions here.

\section{Other applications}

While the present work is aimed at acoustic scattering in the ocean, it also has applicability to other ranging systems and media. A particular example of this is provided by monostatic lidar, ${ }^{29}$ or light detection and ranging, which is being used, inter alia, to characterize the atmospheric surface layer over the sea. ${ }^{30,31}$ In studies of cloud reflectance, for example, the optical analog to Eq. (2) has been recognized. ${ }^{24}$

\section{ACKNOWLEDGMENT}

Dr. D. E. Weston is thanked for his review of the manuscript.

'I. Røttingen, "Distribution and migration of the 1983 year class of Norwegian spring spawning herring in the period July 1987-August 1988," Counc. Meet. Int. Counc. Explor. Sea 1988/H: 41, Copenhagen, Denmark.
${ }^{2} I$. Røttingen, "Reappearance of Norwegian spring spawning herring on spawning grounds south of $60^{\circ} \mathrm{N}$, "Counc. Meet. Int. Counc. Explor. Sea 1989/H: 22, Copenhagen, Denmark.

${ }^{3}$ D. N. MacLennan, "Acoustical measurement of fish abundance," J. Acoust. Soc. Am. 87, 1-15 (1990).

${ }^{4}$ D. E. Weston, "Sound propagation in the presence of bladder fish," in Underwater Acoustics, edited by V. M. Albers (Plenum, New York, 1967), Vol. 2, pp. 55-88.

${ }^{5}$ I. E. Davies, "Attenuation of sound by schooled anchovies," J. Acoust. Soc. Am. 54, 213-217 (1973).

'I. Røttingen, "On the relation between echo intensity and fish density," Fiskeridir. Skr. Ser. Havunders. 16, 301-314 (1976).

${ }^{7}$ M. G. Ertugrul and B. V. Smith, "Multiple scattering effects in fish abundance estimation," Dept. of Electronic and Electrical Engineering Mem. 492 (University of Birmingham, Birmingham, England, 1982).

${ }^{8}$ M. Furusawa, K. Ishii, Y. Miyanohana, and Y. Maniwa, "Experimental investigation of an acoustic method to estimate fish abundance using culture nets," Jpn. J. Appl. Phys. 23 (S23-1), 101-103 (1984).

${ }^{9} \mathrm{~K}$. Olsen, "Sound attenuation within schools of herring," Counc. Meet. Int. Counc. Explor. Sea 1986/B: 44, Copenhagen, Denmark.

${ }^{10}$ F. Armstrong, E. J. Simmonds, and D. N. MacLennan, "Sound losses through aggregations of fish," Proc. IOA 11 (3), 35-43.

"D. N. MacLennan, F. Armstrong, and E. J. Simmonds, "Further observations on the attenuation of sound by aggregations of fish," Proc. IOA 12 (1), 99-106 (1990).

${ }^{12} \mathrm{~K}$. G. Foote, "Analysis of empirical observations on the scattering of sound by encaged aggregations of fish," Fiskeridir. Skr. Ser. Havunders. 16, 423-456 (1978).

${ }^{13}$ K. G. Foote, "Linearity of fisheries acoustics, with addition theorems," J. Acoust. Soc. Am. 73, 1932-1940 (1983).

${ }^{14}$ D. W. Lytle and D. R. Maxwell, "Hydroacoustic assessment in high density fish schools," FAO Fish. Rep. 300, 157-171 (1983).

${ }^{15} \mathrm{~J}$. D. Watson and R. Meister, "Ultrasonic absorption in water containing plankton in suspension," J. Acoust. Soc. Am. 35, 1584-1589 (1963).

${ }^{16}$ W. M. Hamner, P. P. Hamner, S. W. Strand, and R. W. Gilmer, "Behaviour of Antarctic krill, Euphausia superba: chemoreception, feeding, schooling, and molting," Nature 220, 433-435 (1983).

${ }^{17}$ C. H. Greene, P. H. Wiebe, J. Burczynski, and M. J. Youngbluth, "Acoustical detection of high-density krill demersal layers in the submarine canyons off Georges Bank," Science 241, 359-361 (1988).

${ }^{18} \mathrm{~T}$. K. Stanton, "Multiple scattering with applications to fish-echo processing," J. Acoust. Soc. Am. 73, 1164-1169 (1983).

${ }^{19}$ T. K. Stanton, R. D. M. Nash, R. L. Eastwood, and R. W. Nero, "A field examination of acoustical scattering from marine organisms at $70 \mathrm{kHz}$," IEEE J. Ocean Eng. 12, 339-348 (1987).

${ }^{20} \mathrm{C}$. S. Clay and H. Medwin, Acoustical Oceanography: Principles and Applications (Wiley, New York, 1977).

${ }^{21}$ R. J. Urick, Principles of Underwater Sound (McGraw-Hill, New York, 1983), 3rd ed.

${ }^{22}$ J. B. Hersey and R. H. Backus, "Sound scattering by marine organisms," in The Sea, edited by M. N. Hill (Wiley, New York, 1962), Chap. 13, pp. 498-539.

${ }^{23}$ R. B. Mitson, Fisheries Sonar (Fishing News Books, Farnham, England, 1983).

${ }^{24}$ R. C. Anderson and E. V. Browell, "First- and second-order backscattering from clouds illuminated by finite beams," Appl. Opt. 11, 1345-1351 (1972).

${ }^{25}$ H. Bodholt, H. Nes, and H. Solli, “A new echo-sounder system," Proc. IOA 11 (3), 123-130 (1989).

${ }^{26} \mathrm{H}$. P. Knudsen, "Bergen echo integrator: an introduction," J. Cons. Int. Explor. Mer (in press).

${ }^{27}$ K. G. Foote, H. P. Knudsen, R. J. Korneliussen, P. E. Nordb $\phi$, and K. Røang, "Postprocessing system for echo sounder data" J. Acoust. Soc. Am. (to be published).

${ }^{28} \mathrm{~K}$. G. Foote, "On multiple scattering in fisheries acoustics," Counc. Meet. Int. Counc. Explor. Sea 1982/B: 38, Copenhagen, Denmark.

${ }^{29} \mathrm{C}$. F. Bohren and D. R. Huffman, Absorption and Scattering of Light by Small Particles (Wiley, New York, 1983).

${ }^{30} \mathrm{G}$. de Leeuw, "Modeling of extinction and backscatter profiles in the marine mixed layer," Appl. Opt. 28, 1356-1359 (1989).

${ }^{31} \mathrm{G}$. de Leeuw, "Profiling of aerosol concentrations, particle size distributions and relative humidity in the atmospheric surface layer over the North Sea," Tellus 42 B (4) (1990) (in press). 